\title{
Intraoperative Awakening from Endotracheal General Anesthesia for Brain Mapping with Tracheal Tube In Situ
}

\author{
Parmod K. Bithal ${ }^{1} \quad$ Shahenda S. Abdalla ${ }^{1}$ Ravees Jan ${ }^{1} \quad$ Vandan D. Ward ${ }^{1}$ \\ ${ }^{1}$ Department of Anesthesiology and Perioperative Medicine, \\ Address for correspondence Parmod Kumar Bithal, MD, Department of \\ King Fahad Medical City, Riyadh, Kingdom of Saudi Arabia

\begin{abstract}
Anesthesiology and Perioperative Medicine, King Fahad Medical City, PO Box 59046, Riyadh 11525, Kingdom of Saudi Arabia
\end{abstract} \\ (e-mail: bithal.parmod@gmail.com).
}

J Neuroanaesthesiol Crit Care 2021;8:133-135.

\begin{abstract}
Awake craniotomy (AC) is indicated to excise a lesion close to an eloquent area of the brain. Success of this procedure depends upon the patient's active participation during the awake phase of the surgery, especially for brain mapping. Occasionally, a patient may refuse to remain awake during the surgical procedure and demand general anesthesia (GA). A 27-year-old male with uncontrolled seizures from recurrent brain tumor near motor area refused to consent for $A C$ citing his past unpleasant experience; so, the decision to administer GA was taken. To avoid straining/coughing on tracheal tube, his airway was anesthetized with transtracheal xylocaine, bilateral superior laryngeal nerve block, and inflation of tracheal tube cuff with xylocaine. GA was maintained with sevoflurane, infusion of fentanyl, and rocuronium. To awaken him, anesthetics were discontinued and rocuronium antagonized with sugammadex.

Keywords

- awake craniotomy

- airway anesthesia

- brain mapping

- electrocorticography

- eloquent area Intravenous lignocaine and midazolam were administered to supress cough reflex and produce amnesia, respectively. He tolerated the entire duration of 30 minutes of brain mapping with electrocorticography and neurological testing comfortably. Upon completion of brain mapping, GA was reintroduced and the lesion excised. The surgical outcome was good with no neurological deficit. When interviewed postoperatively, the patient had no recall of the awake phase.
\end{abstract}

\section{Introduction}

Awake craniotomy (AC) permits mapping of eloquent areas of the brain, thereby facilitating optimal lesion resection without producing new neurological deficit. Success of this procedure depends on active participation of the patient. Despite the widespread use of this surgical technique, its optimal anesthetic management remains a challenge. ${ }^{1}$ Most anesthesiologists practice asleep-awake-asleep (AAA) technique, without any airway maintenance device. However, many practitioners provide general anesthesia (GA) with airway maintenance devices like laryngeal mask airway (LMA) or nasopharyngeal airway (NPA). The airway device is removed during the awake phase

Published online June 1, 2020
DOI https://doi.org/ 10.1055/s-0040-1710409 ISSN 2348-0548. to avoid patient straining on it during neurological testing and for verbalization of patient in case of speech/language testing. Significantly, these devices do not ensure airway safety, especially if surgery is conducted in an abnormal position.

Unsecured airway is a potential source of ventilation complications. ${ }^{2}$ Such serious issues may arise either due to unintended deep sedation during AAA technique or from administration of sedatives/hypnotic for controlling generalized seizures. The occurrence of seizures is always a possibility in these cases. In such circumstances, the patient requires emergent tracheal intubation. ${ }^{3}$ Securing the lost airway or reinsertion of airway device after completion of brain mapping/lesion resection is extremely difficult because of

(c) 2020. Indian Society of Neuroanaesthesiology and Critical Care.

This is an open access article published by Thieme under the terms of the Creative Commons Attribution-NonDerivative-NonCommercial-License, permitting copying and reproduction so long as the original work is given appropriate credit. Contents may not be used for commercial purposes, or adapted, remixed, transformed or built upon. (https://creativecommons.org/licenses/by-nc-nd/4.0/).

Thieme Medical and Scientific Publishers Pvt. Ltd. A-12, 2nd Floor, Sector 2, Noida-201301 UP, India 
immobility of head and neck in a pinion head holder. This may jeopardize the patient's safety apart from compromising the sterility of the surgical field.

We present a case report of a patient scheduled to undergo $\mathrm{AC}$ for intraoperative brain mapping and resection of a recurrent lesion close to motor strip. This patient refused $A C$ and demanded GA because of his past unpleasant experience of AC.

\section{Case Report}

A 27-year-old, otherwise healthy, male weighing $85 \mathrm{~kg}$, known case of recurrent right frontal oligoastrocytoma abutting the motor strip, presented with the history of tonic clonic seizures. He had undergone AC for the resection of the same lesion 4 years ago and remained seizure free for 3 years. However, from the past 1 year the patient was experiencing frequent seizures daily which were refractory to three antiepileptic drugs.

To preserve motor strip, neurosurgical plan was neuronavigation and magnetic resonance image (MRI) guided AC. However, the patient refused AC citing his past nightmarish experience. Therefore, it was decided to administer general endotracheal anesthesia and awaken him for neurologic examination during brain mapping, without removing the endotracheal tube. He was counseled for the same during the preanesthesia period and advised to remain calm and cooperative during the awake phase.

Anesthesia was induced with fentanyl and propofol, and muscle relaxation was achieved with rocuronium. However, before tracheal intubation, transtracheal $4 \%$ xylocaine $3 \mathrm{ml}$ was injected. Following intubation, cuff was inflated with $6 \mathrm{ml}$ of $2 \%$ plain xylocaine instead of air. In addition, bilateral superior laryngeal nerve block was performed with xylocaine $2 \%$ containing adrenaline. Anesthesia was maintained with oxygen in air, sevoflurane, and infusions of rocuronium and fentanyl. Besides routine ASA monitoring, invasive blood pressure monitoring was also done.

Approximately 10 minutes before the opening of dura, infusions as well as sevoflurane were discontinued. Rocuronium was antagonized with sugammadex and the patient received $3 \mathrm{mg}$ midazolam and $100 \mathrm{mg}$ xylocaine intravenously (IV). When the patient became awake, the neurosurgeon performed the brain mapping, while the patient's motor power in the left upper and lower limb was continuously tested with the patient's cooperation. The mapping was completed in 30 minutes and he tolerated the endotracheal tube during this time without coughing or straining. GA was reintroduced after completion of brain mapping. At the end of the surgery, residual effect of rocuronium was reversed with sugammadex and trachea was extubated (after aspirating residual xylocaine from the cuff). The whole procedure lasted for approximately 7.5 hours. The patient was interviewed next day and he had no recall of the awake period.

\section{Discussion}

Awakening a patient with tracheal tube in situ in the presence of an open skull may be hazardous because of coughing/ straining by the patient, and that may result in brain swelling. Furthermore, even minimal head movement would also be a risk, with loss of neuronavigation. An MRI-compatible pinion head holder does not completely prevent head movements, unlike the Mayfield head holder. The pins of latter hold the head tightly at $60 \mathrm{lbs} / \mathrm{in}^{2}$ of pressure on the skull in an adult. However, pins of an MRI-compatible head holder exert a weaker pressure on the skull compared with the Mayfield head holder and an uncooperative patient can still move his/her head. Therefore, as a rule, surgery of lesions of eloquent areas of brain is performed in an awake state, with the patient counseled preoperatively, to avoid onset of new postoperative neurologic deficit. Despite the benefits of AC, sometimes the awake procedure has to be converted to GA due to various reasons. ${ }^{3,4}$

The AC under sedation (AAA) technique results in higher incidences of hemodynamic disturbances versus the GA techniques. ${ }^{5}$ The technique of GA with LMA may be an acceptable choice but can cause delayed awakening, dull response, or respiratory depression from residual anesthetic effects after extubation. ${ }^{6}$ Additionally, LMA fails to prevent aspiration of gastric contents. ${ }^{7}$ LMA also risks dislodgement in an abnormal position surgery and its reinsertion after completion of brain mapping/lesion resection in a fixed head is not easy and requires considerable expertise. Reinsertion of any airway device would necessitate removal of the sterile drapes, thereby disrupting the sterility of the surgical field. Anesthesia management with NPA for AC is associated with incidences of hypoxia and hypercarbia. ${ }^{8}$ Importantly, both of these airway devices are unsafe in presence of obesity or obstructive sleep apnea.

Since an awake/unanesthetized patient cannot tolerate the ET, we anesthetized the airway with transtracheal administration of $4 \%$ xylocaine and blocking the superior laryngeal nerve, bilaterally. This technique of anesthetizing the airway is frequently employed for awake fiberoptic tracheal intubation to suppress the cough reflex. To further enhance tolerance to ET we inflated the cuff with xylocaine, and administered xylocaine intravenously as well. ${ }^{9,10} \mathrm{~A}$ small dose ( $3 \mathrm{mg}$ ) of midazolam was given IV to achieve amnesia during awake state. ${ }^{11}$ Such a small dose of midazolam does not interfere with acquisition of electrocorticography (ECoG) signals. ${ }^{12}$ Our patient remained fully cooperative during the neurological testing, without straining/coughing or making any undesirable movements. He had no obvious discomfort, and had good surgical outcome without any new neurological deficit.

At least $6.4 \%$ awake craniotomies need conversion to GA. ${ }^{13}$ Our technique of combining airway block and GA will be beneficial in apprehensive patients requiring brain mapping procedures and do not need verbalization by the patient. However, our technique cannot be used for resection 
of lesions adjacent to speech/language areas which require patient to converse with the neurosurgeon.

\section{Conclusion}

General endotracheal anesthesia in combination with airway anesthesia to facilitate ET tolerance during the awake state along with midazolam and preoperative counseling is a suitable alternative to AC. This is especially beneficial in an apprehensive patient who demands GA but whose pathology otherwise has a strong indication for AC for brain mapping for optimal resection of lesion.

\section{Conflict of Interest}

None declared.

\section{References}

1 Taylor MD, Bernstein M. Awake craniotomy with brain mapping as the routine surgical approach to treating patients with supratentorial intraaxial tumors: a prospective trial of 200 cases. J Neurosurg 1999;90(1):35-41

2 Sinha PK, Koshy T, Gayatri P, Smitha V, Abraham M, Rathod RC. Anesthesia for awake craniotomy: a retrospective study. Neurol India 2007;55(4):376-381

3 Blanshard HJ, Chung F, Manninen PH, Taylor MD, Bernstein M. Awake craniotomy for removal of intracranial tumor: considerations for early discharge. Anesth Analg 2001;92(1):89-94

4 Piccioni F, Fanzio M. Management of anesthesia in awake craniotomy. Minerva Anestesiol 2008;74(7-8):393-408
5 Skucas AP, Artru AA. Anesthetic complications of awake craniotomies for epilepsy surgery. Anesth Analg 2006;102(3): 882-887

6 Chung YH, Park S, Kim WH, Chung IS, Lee JJ. Anesthetic management of awake craniotomy with laryngeal mask airway and dexmedetomidine in risky patients. Korean J Anesthesiol 2012;63(6):573-575

7 Deras P, Moulinié G, Maldonado IL, Moritz-Gasser S, Duffau $\mathrm{H}$, Bertram L. Intermittent general anesthesia with controlled ventilation for asleep-awake-asleep brain surgery: a prospective series of 140 gliomas in eloquent areas. Neurosurgery 2012;71(4):764-771

8 Sivasankar C, Schlichter RA, Baranov D, Kofke WA. Awake craniotomy: a new airway approach. Anesth Analg 2016;122(2):509-511

9 Huang CJ, Tsai MC, Chen CT, Cheng CR, Wu KH, Wei TT. In vitro diffusion of lidocaine across endotracheal tube cuffs. Can J Anaesth 1999;46(1):82-86

10 Poulton TJ, James FM III. Cough suppression by lidocaine. Anesthesiology 1979;50(5):470-472

11 Koyama S, Ohashi N, Kurita S, Nakatani K, Nagata N, Toyoda Y. Conscious sedation and amnesic effect of intravenous lowdose midazolam prior to spinal anesthesia [in Japanese]. Masui 2008;57(6):713-718

12 Formaggio E, Storti SF, Tramontano V, et al. Frequency and time-frequency analysis of intraoperative ECoG during awake brain stimulation. Front Neuroeng 2013;6:1-8

13 Nossek E, Matot I, Shahar T, et al. Failed awake craniotomy: a retrospective analysis in 424 patients undergoing craniotomy for brain tumor. J Neurosurg 2013;118(2):243-249 